\title{
Tratamiento de la maloclusión clase III con máscara facial y hyrax híbrido, protocolo Alt-RAMEC
}

\author{
Treatment of Class III Malocclusion with Facial Mask, \\ Hybrid Hyrax and Alt-RAmec Protocol
}

Jesús Alberto Hernández Silva $\bowtie$, Esp. ${ }^{1}$, Carolina Rodríguez Manjarrés, Esp. ${ }^{2}$, José Manuel Martínez Ávila, Odon. ${ }^{3}$

\author{
${ }^{1}$ Universidad del Valle, Cali, Colombia \\ 2 Universidad del Valle, Cali, Colombia \\ ${ }^{3}$ Universidad del Valle, Cali, Colombia
}

Escuela de Odontología, Facultad de Salud, Universidad del Valle, Calle 3 n. ${ }^{\circ} 36 B-00$, Edificio 132, San Fernando, Cali, Colombia. Correo electrónico: sualberto@msn.com

Recibido: 16 de febrero del 2016 Aprobado: 7 de abril del 2016

Cómo citar este artículo: Hernández-Silva JA, Rodríguez-Manjarrés C, Martínez-Ávila JM. Tratamiento de la maloclusión clase III con máscara facial y hyrax híbrido, Protocolo Alt-ramec. Rev Nac Odontol. 2018;13(26):1-9. doi: http://dx.doi. org/10.16925/od.v13i26.2045

\section{Resumen}

Introducción: las dificultades en la corrección de las discrepancias óseas que presentan las maloclusiones clase III debido a la complejidad de los tratamientos existentes, lo impredecibles que son el crecimiento óseo, el desarrollo y los resultados de tratamiento para los pacientes jóvenes, sumado a las características físicas evidentes en los sujetos que las padecen, hacen que sean todo un reto a la hora de plantear planes de tratamiento exitosos para su corrección.

Caso: paciente masculino, 10 años de edad, asiste para reevaluación a la clínica de ortopedia maxilar del posgrado de Odontología Pediátrica y Ortopedia Maxilar de la Escuela de Odontología de la Universidad del Valle (Cali-Colombia). No refiere antecedentes familiares de anomalías esqueléticas o dentarias; clínicamente se observa un mentón prominente.

Objetivo: evaluar el efecto del tratamiento en un paciente, manejado con hyrax híbrido, máscara de protracción y miniimplantes para la corrección de la maloclusión clase l..

Resultados y conclusiones: la combinación de hyrax híbrido, máscara facial y protocolo Alt-RAmec responde a un cambio esquelético real por la transmisión de fuerzas directamente al hueso, con lo que se abolen los camuflajes dentoalveolares que generan resultados satisfactorios a nivel dental, facial e incluso psicológico en pacientes con clase II esquelética.

Palabras clave: Alt-RAmEc, hyrax híbrido, maloclusión clase II, máscara facial, miniimplantes. 


\title{
Treatment of Class III Malocclusion with Facial Mask, Hybrid Hyrax and Alt-RAMEc Protocol
}

\begin{abstract}
Introduction: The difficulties in correcting the bone discrepancies of class III malocclusions due to the complexity of existing treatments, the unpredictability of bone growth, the development and treatment outcomes for young patients, and the evident physical characteristics of the subjects who suffer from them make them a challenge when considering successful treatment plans for correction.

Case: A 10-year-old male patient attended the maxillary orthopedics clinic of the graduate program Pediatric Dentistry and Maxillary Orthopedics of the School of Dentistry at the Universidad del Valle (Cali-Colombia) for re-evaluation. He does not report family history of skeletal or dental anomalies; a prominent chin is clinically observed.

Aim: To assess the effect of a treatment with hybrid hyrax, protraction mask and mini-implants in a patient for the correction of class III malocclusion.

Results and conclusions: The combination of hybrid hyrax, face mask and Alt-RAmec protocol responds to a real skeletal change due to the direct transmission of forces to the bone, thus abolishing the dentoalveolar camouflages that produce satisfactory results at the dental, facial and even psychological levels in patients with class II skeletal malocclusion.
\end{abstract}

Keywords: Alt-RAmEc, hybrid hyrax, class III malocclusion, facial mask, mini-implants.

\section{Tratamento da maloclusão classe III com máscara facial e Hyrax híbrido, protocolo Alt-RAMEC}

\begin{abstract}
Resumo
Introdução: as dificuldades na correção das discrepâncias ósseas que apresentam as maloclusões classe III devido à complexidade dos tratamentos existentes, ao imprescindível que é o crescimento ósseo, ao desenvolvimento e resultados de tratamento para os pacientes jovens, somado às características físicas evidentes nos sujeitos que as padecem, fazem com que ela seja um desafio na hora de propor planos de tratamento bem-sucedidos para sua correção.

Caso: paciente masculino, 10 anos de idade, consulta para reavaliação na clínica de ortopedia maxilar da pós-graduação de Odontologia Pediátrica e Ortopedia Maxilar da Escola de Odontologia da Universidad del Valle (Cali, Colômbia). Não refere antecedentes familiares de anomalias esqueléticas ou dentárias; clinicamente, observa-se um queixo proeminente.

Objetivo: avaliar o efeito do tratamento num paciente, administrado com Hyrax híbrido, máscara de protoação e mini-implantes para a correção da maloclusão classe I..

Resultados e conclusões: a combinação de Hyrax híbrido, máscara facial e protocolo Alt-RAmEc responde a uma mudança esquelética real pela transmissão de forças diretamente ao osso, com o que se abolem as camuflagens dentoalveolares que geram resultados satisfatórios no âmbito dental, facial e inclusive psicológico em pacientes com classe III esquelética.
\end{abstract}

Palavras-chave: Alt-RAMEc, Hyrax híbrido, maloclusão classe II, máscara facial, mini-implantes. 


\section{Introducción}

La maloclusión clase III se caracteriza por una relación mesial de la mandíbula, tomando como referencia la cúspide mesovestibular del primer molar que ocluye mesial al surco del primer molar inferior $[1,2]$. Puede ser debido a retrusión del maxilar, prognatismo mandibular o ambas [3].

La incidencia de maloclusiones clase III difiere entre los diferentes grupos étnicos [4]; los estudios epidemiológicos reportan prevalencia entre 1 y $14 \%[3,5]$. Parece ser común sobre todo en los de ascendencia asiática (prevalencia en población china puede ser mayor al $12 \%$ ) y menos prevalente en la población europea (1,5 a 5,3\%). En poblaciones norteamericanas caucásicas, el rango varía de 1 a $4 \%$ [6].

En Colombia, dos estudios reportan prevalencia asociada a esta maloclusión. Según el Iv Estudio Nacional de Salud Bucal-ensab IV, en niños de 5 años se encontró una prevalencia del 2,41\%, y en la población de 12 años, del 3,83\% [7]. Por otra parte, en el estudio de Thilander et al. [8], se encontró una prevalencia de mordida cruzada anterior, que en la mayoría de los casos es una manifestación de una maloclusión clase iII del 5,8\% en una población entre 5 y 17 años de edad, y la maloclusión clase III propiamente definida del 3,7\%.

Su etiología es multifactorial, aunque presenta una fuerte base genética, siendo el resultado de una interacción genética y ambiental $[9,10]$. Puede tener combinaciones de componentes esqueléticos, dentoalveolares y/o funcionales en los tres planos del espacio transversal, sagital y vertical $[9,11,12]$.

Cuando presenta un componente esquelético, está asociado con discrepancias en el tamaño y/o posición de los maxilares, en las cuales la herencia juega un papel importante, sobre todo en casos de prognatismo mandibular $[13,14]$.

Cuando es de tipo dentoalveolar, está favorecida por factores como: patrón de erupción lingual y/o erupción retardada de los dientes incisivos superiores, inclinación vestibular de incisivos inferiores, presencia de dientes supernumerarios $\mathrm{u}$ odontomas y longitud inadecuada del arco dental, entre otros $[13,14]$. También puede ser producto de hábitos de mala postura mandibular, respiración oral o posición de la lengua adelantada y aplanada, en lo cual a veces no se puede determinar con exactitud si la función alterada de la lengua es un fenómeno adaptativo a un factor etiológico primario $[14,15]$.
Si es una maloclusión pseudoclase iII o tipo funcional, la mandíbula se posiciona en cierre en una posición anterior respecto al maxilar superior. Este movimiento es, por lo general, el resultado de contactos dentales prematuros que provocan ese desplazamiento [15].

Hoy en día, se aceptan los beneficios del tratamiento temprano para la corrección de la maloclusión clase III, cuyos objetivos están direccionados a restringir el crecimiento mandibular, estimular el desarrollo del maxilar superior, recuperar fisiología de la ATM, evitar fuerzas oclusales traumáticas sobre los dientes y el periodonto, lograr la corrección del overjet y mejorar postura labial y facial, entre otros [14,16].

Entre las opciones de tratamiento encontramos aparatología ortopédica funcional, fija o mecánica extraoral. En el caso específico de la maloclusión clase III con deficiencia de los maxilares, el tratamiento incluye el uso de máscara facial y disyunción maxilar, la cual puede elaborarse con aparatología tipo hyrax con férula de McNamara o soldado a bandas [14,17].

Algunos autores reportaron efectos indeseables como el movimiento dental no deseado (migración mesial) o problemas periodontales con el uso de máscara facial y expansor tipo hyrax; para evitar esto y transferir la fuerza directamente al hueso maxilar, se han diseñado protocolos de anclaje como los implantes dentales y microimplantes quirúrgi$\cos [18,19]$.

Wilmes [18] et al. introdujeron el hyrax híbrido utilizando miniimplantes en la zona anterior del paladar para proporcionar anclaje. Esto combinado con máscara facial de protracción potenciará los efectos esqueléticos del tratamiento. Los autores proponen realizar expansión rápida maxilar alternada con constricción (cierre del tornillo), protocolo Alt-RAMEC, planteado por Eric Liou para mantener la estimulación sutural por un periodo de tiempo, con lo que se alcanza una mayor protracción maxilar [18].

El protocolo comprende siete a ocho semanas, se emplea un hyrax híbrido soldado a bandas a nivel de segundos molares temporales o primer molar permanente, y un anclaje óseo a través de dos miniimplantes ubicados en la zona anterior del paladar para disminuir los efectos indeseables mencionados. El hyrax es activado $1 \mathrm{~mm}$ (cuatro vueltas) por día, alternando una semana de expansión con otra de constricción. Se emplea una 
máscara facial y elásticos intraorales, que generan $400 \mathrm{~g}$ de fuerza de protracción a cada lado para la corrección de la maloclusión $[18,20,21]$.

En este artículo, se reportan los efectos de la terapia descrita en un paciente masculino, de 10 años, con maloclusión clase III esquelética severa y dentición mixta tardía. Se analizan los cambios clínicos y radiográficos después de diez meses de empleado el protocolo.

\section{Reporte de caso}

Paciente masculino, 10 años de edad. Asiste para reevaluación a la clínica de ortopedia maxilar del posgrado de Odontología Pediátrica y Ortopedia Maxilar de la Escuela de Odontología de la Universidad del Valle (Cali, Colombia). Motivo de consulta referido por su madre: "Necesito volver a empezar un tratamiento con mi hijo porque está muy mal, tiene la mandíbula muy grande y no quiero que lo operen".

Paciente procedente de la ciudad de Cali (Colombia), estrato socioeconómico 3. Al revisar la historia clínica, no se encontró ningún tipo de compromiso sistémico, ni antecedentes médicos de importancia. Ausencia de hábitos. No refiere antecedentes familiares de anomalías esqueléticas o dentarias; clínicamente se observa un mentón prominente que han notado familiares y el paciente, quienes refieren que debido a la posición adelantada de la mandíbula, la apariencia facial se ve afectada.

Dentro del protocolo de atención, se solicitaron las siguientes ayudas diagnósticas: fotografías intraorales y extraorales, modelos de estudio, y radiografía panorámica y lateral de cráneo.

Examen clínico extraoral: paciente de raza mestiza, cráneo dolicocefálico, biotipo facial leptoprosopo. El examen de las proporciones faciales verticales reveló el tercio facial inferior aumentado, incompetencia labial, asimetría facial, línea media dental superior no coincidente con línea media facial. Perfil cóncavo (Figura 1).

Examen miofuncional: respirador nasal, deglución con protrusión lingual, desviación mandibular hacia la derecha, ausencia de dolor y ruidos a nivel de la articulación temporomandibular.

Examen clínico intraoral: presenta un estado de higiene oral bueno. Presenta mordida abierta anterior de $3 \mathrm{~mm}$ entre dientes 11, 12, 41, 42 y 83, mordida cruzada anterior entre 11-41, 12-42, 21-31 y 32, mordida cruzada posterior unilateral derecha. Relación molar clase III bilateral, relación canina izquierda clase III, lado derecho no aplica por ausencia de canino superior. Relación molar clase III bilateral, relación canina izquierda clase III, lado derecho no aplica por ausencia de canino superior, overjet $-6 \mathrm{~mm}$, overbite del $0 \%$. Desviación de línea media dental $3 \mathrm{~mm}$ a la derecha. Arcos dentales en forma parabólica, asimétricos. Rugas palatinas pronunciadas, paladar profundo. Dentición mixta, proinclinación dientes anteriores inferiores, apiñamiento anteroinferior, diente 53 ausente y $13 \mathrm{sin}$ erupcionar (Figura 2).

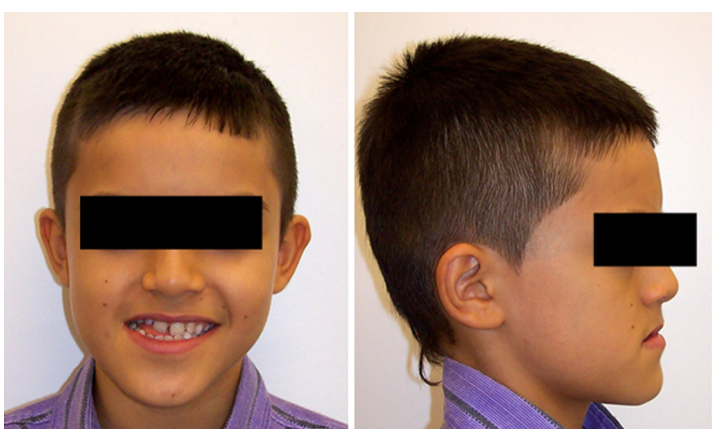

Figura 1. Fotografías extraorales pretratamiento: frente y perfil Fuente: elaboración propia
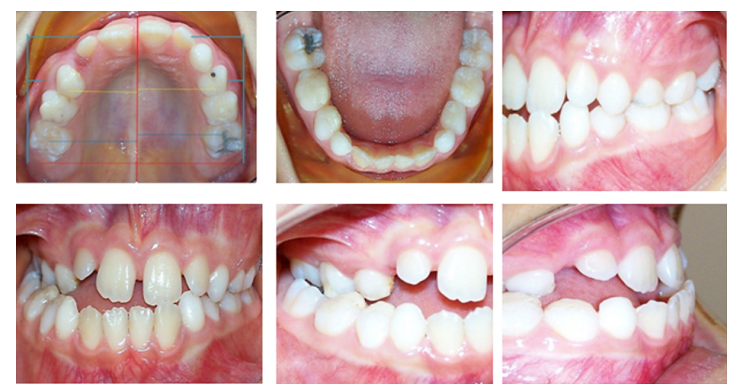

Figura 2. Análisis intraoral

Fuente: elaboración propia

Análisis radiográfico: en la radiografía panorámica, se observa tabique nasal derecho desviado hacia la izquierda y cornete derecho hipertrófico, paladar asimétrico, con evidente descenso derecho. Cóndilo izquierdo levemente delgado respecto al derecho. Cuerpo mandibular asimétrico, con mayor tamaño de hemimandíbula izquierda, compatible con desviación del mentón clínico. 
Dentición mixta, presencia de 12 dientes permanentes en boca, gérmenes aún en proceso de formación. Se observan dientes 13 y 23 con falta de espacio aparente para su correcta erupción. No se observan anomalías dentales de forma, tamaño o posición (Figura 3).

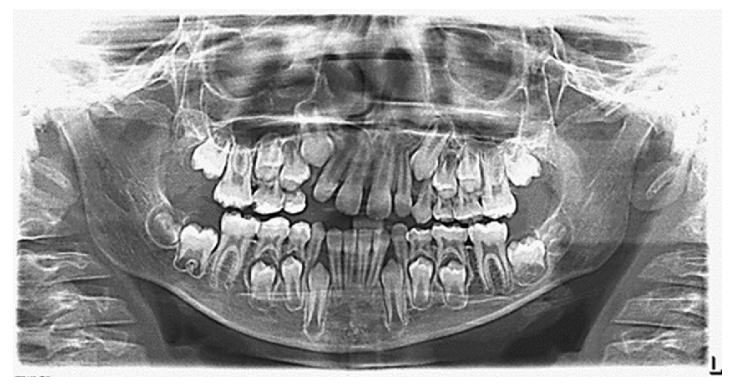

Figura 3. Radiografía panorámica inicial Fuente: elaboración propia

Análisis esquelético: micrognatismo mandibular, retrognatismo maxilar (SNA $77^{\circ}$ ), SNB $80^{\circ}$, clase III esquelética, dolicofacial, crecimiento rotacional posterior. Protrusión y proinclinación de incisivos superiores e inferiores (Figura 4).

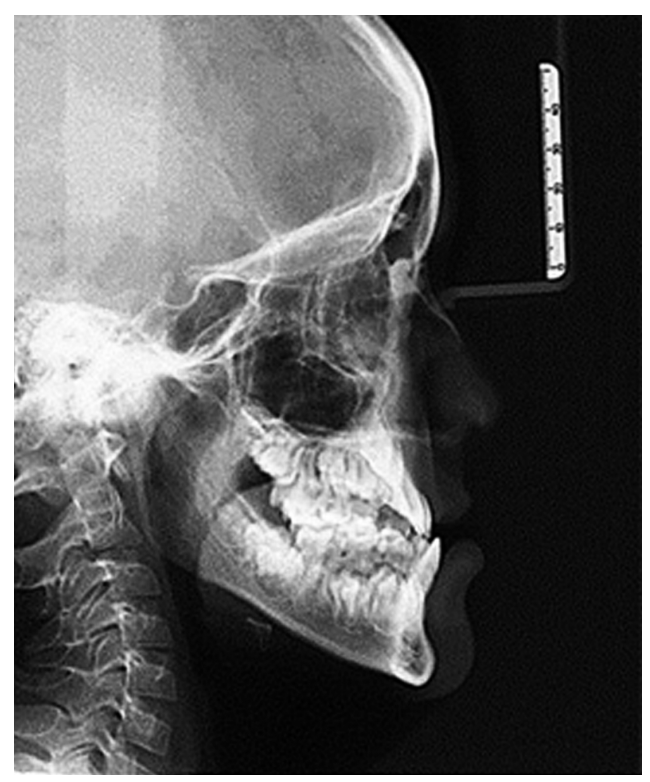

Figura 4. Radiografía lateral de cráneo inicial Fuente: elaboración propia
Otros valores de interés: Wits -9mm, Go-Me 85 $\mathrm{mm}$ (aumento en longitud del cuerpo mandibular), Ena-Me $71 \mathrm{~mm}$ (aumento en el 1/3 inferior facial).

Diagnóstico: paciente de 10 años, género masculino, con dentición mixta; clase III esquelética por retrognatismo maxilar. Presenta mordida cruzada anterior y posterior unilateral derecha y mordida abierta anterior.

\section{Objetivos del tratamiento}

Fase 1: estimular el crecimiento transversal del maxilar para recuperar espacio de caninos permanentes, promover el crecimiento anterior del maxilar y restringir el crecimiento mandibular excesivo y cambiar la dirección de crecimiento.

Fase 2: una vez concluida la fase 1, se remitirá a la clínica de posgrado de Ortodoncia de la Universidad del Valle para reevaluación del caso e inicio de tratamiento con aparatología ortodóncica fija.

Antes de iniciar el tratamiento, los acudientes del menor firmaron el consentimiento informado.

\section{Plan de tratamiento}

Se seleccionó el uso de aparatología fija tipo hyrax híbrido soldado a bandas a nivel de primeros molares permanentes superiores con ganchos vestibulares para combinar con máscara de protracción maxilar (400 g de fuerza a cada lado) y protocolo Alt-RAMEC. Se emplearon dos microimplantes marca Dentos, posicionados a nivel de las segundas rugas palatinas, uno a cada lado de la sutura media palatina (Figura 5).

Durante nueve semanas, se realizó el protocolo Alt-RAmec. Se activó el tornillo en casa por el acudiente (mamá), $1 \mathrm{~mm}$ diario (una vuelta) durante la primera semana para realizar expansión rápida maxilar; a la semana siguiente se realizaba constricción maxilar de $1 \mathrm{~mm}$ diario, devolviendo $1 \mathrm{~mm}$ diario el tornillo de expansión, hasta quedar en la posición inicial al finalizar la segunda semana (tornillo completamente cerrado). Se siguió el protocolo clínico según lo propuesto en la literatura: expansión durante las semanas impares y constricción durante las semanas pares (Tabla 1). El uso de la máscara facial de protracción empezó desde la semana dos, indicando al paciente el uso diario durante mínimo 12 horas. 

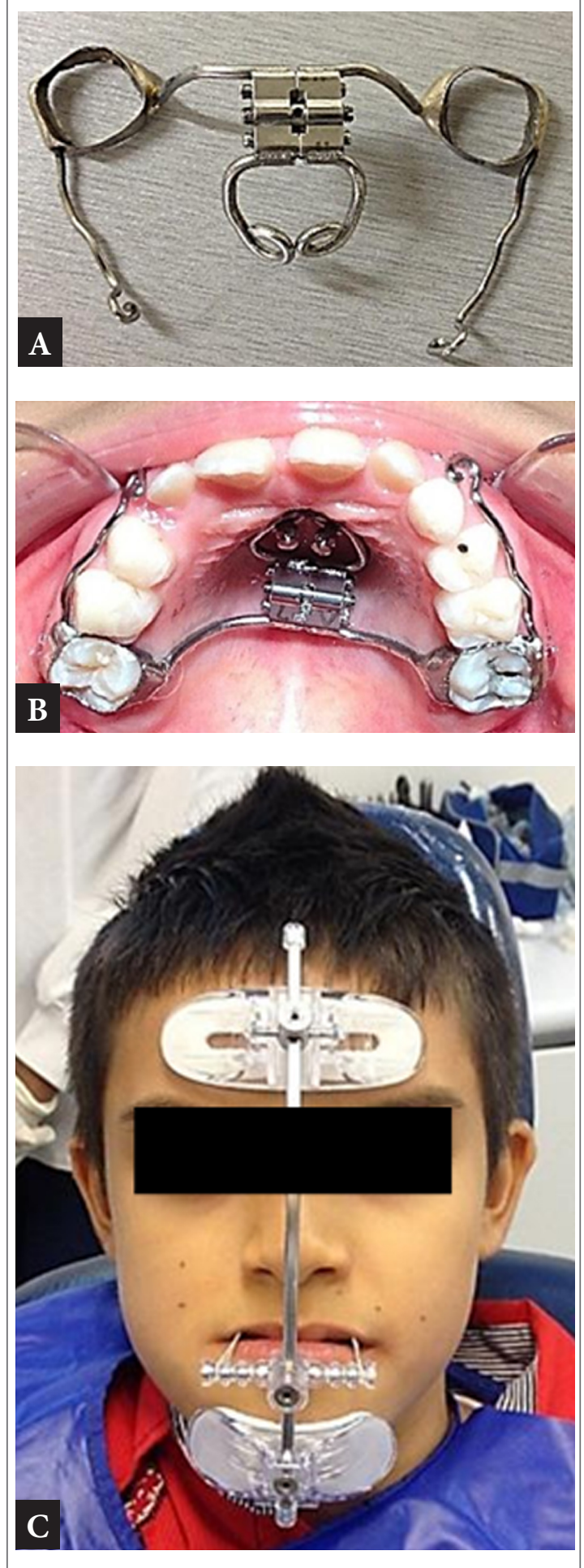

Figura 5. A-B. Hyrax modificado para anclaje óseo directo por medio de microimplantes-máscara facial. C. Paciente con máscara facial

Fuente: elaboración propia
Tabla 1. Protocolo clínico para alternar expansión rápida maxilar y constricción (Alt-RAMEC)

\begin{tabular}{ccc}
\hline $\begin{array}{c}\text { Secuencia } \\
\text { alternativa } \\
\text { semanal }\end{array}$ & $\begin{array}{c}\text { Cantidad semanal } \\
\text { de expansión/ } \\
\text { constricción }\end{array}$ & $\begin{array}{c}\text { Cantidad diaria } \\
\text { de activación }\end{array}$ \\
\hline Expansión & $7 \mathrm{~mm}$ & $1 \mathrm{~mm}$ \\
Constricción & $7 \mathrm{~mm}$ & $1 \mathrm{~mm}$ \\
Expansión & $7 \mathrm{~mm}$ & $1 \mathrm{~mm}$ \\
Constricción & $7 \mathrm{~mm}$ & $1 \mathrm{~mm}$ \\
Expansión & $7 \mathrm{~mm}$ & $1 \mathrm{~mm}$ \\
Constricción & $7 \mathrm{~mm}$ & $1 \mathrm{~mm}$ \\
Expansión & $7 \mathrm{~mm}$ & $1 \mathrm{~mm}$ \\
\hline
\end{tabular}

Fuente: [22]

Evolución del tratamiento: 12 meses después, clínicamente, se observa un overjet de $1,5 \mathrm{~mm}$ y ausencia de mordida cruzada anterior y posterior derecha (Figura 6). Se tomaron radiografías posteriores al tratamiento; en la radiografía panorámica se observa mayor simetría a nivel condilar, paladar presenta inclinación menos evidente que en radiografía previa; a nivel del sector anterior, se observa mayor espacio en sentido transversal y una ruta de erupción más apropiada para diente 13. En la mandíbula, se observa mayor simetría a nivel del mentón (Figura 7).

Sobre la radiografía lateral de cráneo, se realizaron nuevos trazos cefalométricos y se encontró un aumento en el ángulo SNA, valor $82^{\circ}$, ángulo ANB de $1^{\circ}$, Wits de $-1,5 \mathrm{~mm}$, con lo que se logró una corrección de la discrepancia maxilomandibular (Figura 8 y Tabla 2).

Actualmente, el paciente se encuentra en periodo de retención, realizado con el hyrax, el cual se fijó en posición final. Se espera finalización de recambio dental para iniciar tratamiento de ortodoncia, en el cual se utilizará la técnica MEAW.

\section{Discusión}

Varios autores reportan en la literatura el tratamiento temprano para la corrección de la maloclusión clase III $[18,23]$. En este caso, se empleó un tratamiento para comprobar los beneficios de la máscara facial con hyrax híbrido y protocolo Alt-RAmEC antes descrito en un paciente en crecimiento, dentición mixta con maloclusión 

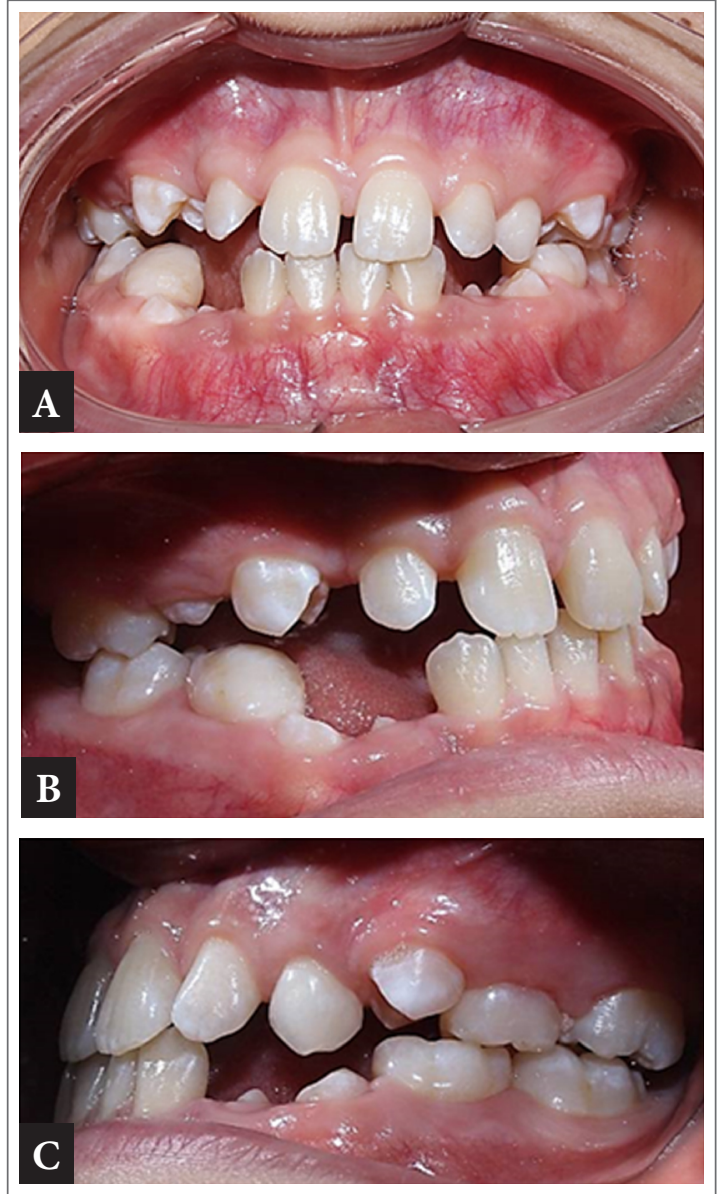

Figura 6. Fotografías intraorales doce meses después de iniciado el tratamiento. A. Frontal. B. Oclusión derecha.

C. Oclusión izquierda

Fuente: elaboración propia

esquelética clase III severa y un pronóstico reservado con tratamiento de ortopedia convencional. La corrección de la discrepancia esquelética aquí observada es similar al promedio en los valores de Wits de 5,7 mm obtenidos por Wilmes et al. [18]

Tabla 2. Valores cefalométricos comparativos del paciente durante el primer año de tratamiento

\begin{tabular}{ccccc}
\hline Puntos & Norma & Inicial & $\mathbf{1}$ año & Diferencia \\
\hline SNA & $80,6^{\circ}+/-3^{\circ}$ & $77^{\circ}$ & $82^{\circ}$ & $5^{\circ}$ \\
ANB & $4,2^{\circ}+/-1,9^{\circ}$ & $3^{\circ}$ & $1^{\circ}$ & $2^{\circ}$ \\
Wits & $+: 1 \mathrm{~mm}$ & $-9 \mathrm{~mm}$ & $-1,6 \mathrm{~mm}$ & $7,4 \mathrm{~mm}$ \\
& $+00 \mathrm{~mm}$ & & & \\
\hline
\end{tabular}

Fuente: elaboración propia

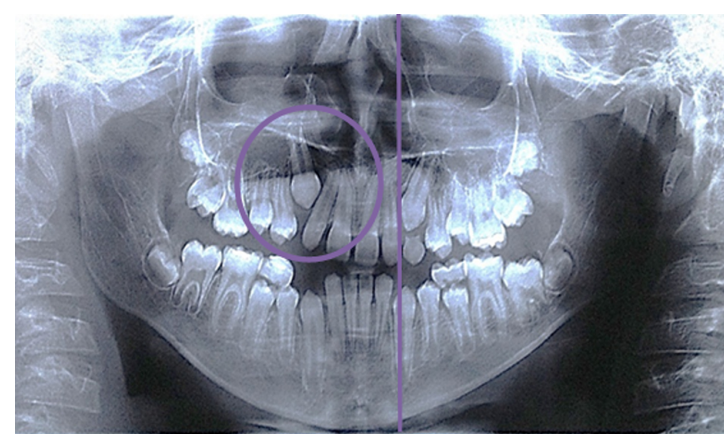

Figura 7. Radiografía panorámica doce meses después de iniciado el tratamiento

Fuente: elaboración propia

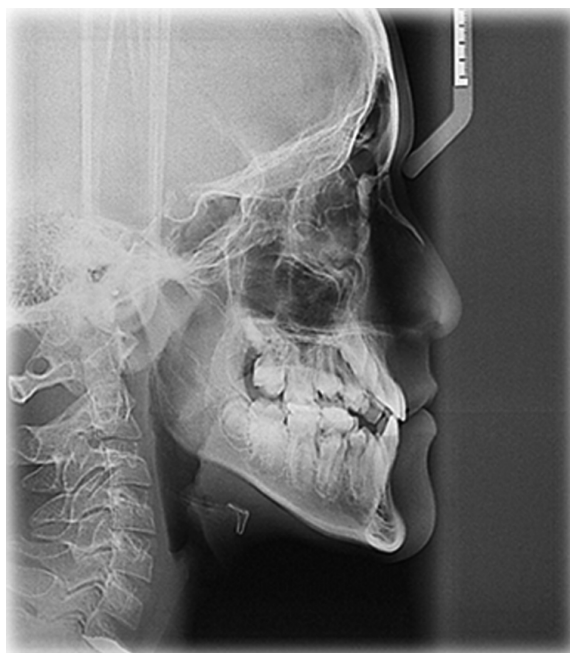

Figura 8. Radiografía lateral de cráneo doce meses después de iniciado el tratamiento

Fuente: elaboración propia

en el 2014, en comparación con 7,5 mm correspondientes al cambio después de un año de tratamiento en este caso.

Teniendo en cuenta los resultados del estudio realizado por Sar et al. en el 2011 [24], quienes implementaron en quince de sus pacientes el protocolo de protracción maxilar con dos miniimplantes de titanio y máscara de protracción, se observa un avance del maxilar de 2,3 $\mathrm{mm}$ después de 6,78 meses de tratamiento; además, mostró rotación anterior del maxilar, rotación posterior de la mandíbula y un ligero aumento de la altura facial, mejorando las relaciones maxilomandibulares y el perfil de los tejidos blandos. De acuerdo con los resultados obtenidos en este caso, se pueden observar similitudes en cuanto a los cambios obtenidos en 
los ángulos SNA y ANB, los cuales inicialmente fueron de $77^{\circ}$ y $3^{\circ}$, respectivamente, y después de un año de iniciado el tratamiento fueron de $82^{\circ}$ (SNA) y $1^{\circ}$ (ANB).

Ngan et al. [25] realizaron un análisis de cambios cefalométricos comparando pacientes tratados con expansión rápida palatina (ERP) y protracción del maxilar con un grupo control, en el que observaron un promedio de cambio en el ángulo sNA de $3^{\circ}$ al finalizar el tratamiento; en este caso, al finalizar el protocolo Alt-RAMEC y la protracción maxilar empleando máscara facial, se obtuvo un cambio de $5^{\circ}$ en el ángulo sNa. Podría entonces confirmarse que se alcanzan mejores resultados al "desarticular” el maxilar con el protocolo Alt-RAMEC y realizar anclaje directamente sobre el hueso para la corrección de las maloclusiones clase III.

El overjet cambió considerablemente. Lo reportado aquí después de 12 meses de iniciado el tratamiento es de $7,5 \mathrm{~mm}$, valor similar al patrón observado en el cambio de la sobremordida horizontal en la mayoría de literatura revisada, siendo este cambio una contribución netamente esquelética [25].

Sin embargo, es importante destacar que aunque existen diferentes terapias para la corrección de una clase III esquelética, debido a la magnitud de la maloclusión aquí expuesta y al periodo de dentición del paciente, se seleccionó este protocolo que mostró resultados efectivos y estables en la fase de observación. Este tratamiento fue llevado a cabo por ortopedistas maxilares y requirió un manejo multidisciplinario de las áreas de periodoncia y ortodoncia.

\section{Conclusiones}

La combinación de hyrax híbrido, máscara facial y protocolo Alt-RAMEC responde a un cambio esquelético real por la transmisión de fuerzas directamente al hueso, con lo que se abolen los camuflajes dentoalveolares que generan resultados satisfactorios a nivel dental, facial e incluso psicológico en pacientes con clase III esquelética.

Se cumplieron los objetivos de tratamiento planteados en la fase 1, se consiguió estimular el crecimiento transversal del maxilar para recuperar espacio de caninos permanentes gracias al protocolo de expansión y constricción maxilar
Alt-RAMEC, a la vez que se evitaron los eventos indeseables como la migración mesial y el daño periodontal.

Se logró promover el crecimiento anterior del maxilar gracias al efecto de la expansión rápida maxilar apoyado en la protracción con la máscara facial. El crecimiento mandibular excesivo y su dirección de crecimiento también se controlaron. Por lo tanto, se concluye que el uso de máscara facial, hyrax híbrido y protocolo Alt-RAMEc otorga una corrección de la discrepancia maxilomandibular propia de pacientes con maloclusión clase in en un periodo de tiempo relativamente corto, gracias al efecto de "desarticulación" que sufre el maxilar, disminuyendo de igual manera el porcentaje de recidiva para estos pacientes.

Se debe investigar mucho más y tener ensayos clínicos aleatorizados con pacientes controles positivos y negativos para poder hacer conclusiones definitivas al respecto, además de estudios prospectivos con los efectos a mediano y a largo plazo para determinar el nivel de recidiva que se presenta en estos casos.

\section{Referencias}

[1] Angle EH. Classification of malocclusion. Dental Cosmos. 1899;41:248-64.

[2] Matheus-Melo P, De Lima-Santos N, Rangel-Barreto dos Santos C, Carvalho-Souza Baião F, Rangel-Pinheiro MC, Matos-Neto M, Andrade-Souza $\mathrm{I}$, et al. Is alternate rapid maxillary expansion and constriction protocol effective in the treatment of Class III malocclusion? A systematic review. Dental Press J Orthod. 2016;21(6):34-42.

[3] Rathi AR, Kumari NR, Vadakkepuriyal K, Santhkumar M. Treatment of skeletal Class III malocclusion using face mask therapy with alternate rapid maxillary expansion and constriction (Alt-RAMEC) protocol. J Indian Soc Pedod Prev Dent. 2015;33:341-3.

[4] Lew KK, Foong WC. Horizontal skeletal typing in an ethnic Chinese population with true Class III malocclusions. Br J Orthod. 1993;20(1):19-23.

[5] Nishitha J, Ahmad H, Walid F. Skeletal malocclusion: A developmental disorder with a life-long morbidity. J Clin Med Res. 2014;6(6):399-408.

[6] Baccetti T, Reyes BC, McNamara JA Jr. Gender differences in Class III malocclusion. Angle Orthod. 2005;75(4):510-20.

[7] Ministerio de Salud de Colombia. Iv Estudio Nacional de Salud Bucal - ENSAB Iv. Bogotá: Ministerio de Salud [Internet]; 2014. Disponible en: https://www. minsalud.gov.co/sites/rid/Lists/BibliotecaDigital/ RIDE/VS/PP/ENT/abc-salud-bucal.pdf. 
[8] Thilander B, Pena L, Infante C, Parada SS, De Mayorga C. Prevalence of malocclusion and orthodontic treatment need in children and adolescents in Bogota, Colombia. An epidemiological study related to different stages of dental development. Eur J Orthod. 2001;23(2):153-67.

[9] Espinar E, Ruiz MB, Ortega H, Llamas JM, Barrera JM, Solano JE. Tratamiento temprano de las clases III. Rev Esp Ortod. 2011;41:79-89.

[10] Jacobson A, Evans WG, Preston CB, Sadowsky PL. Mandibular prognathism. Am J Orthod. 1974; 66(2):140-71.

[11] Rabie AB, Gu Y. Diagnostic criteria for pseudoClass III malocclusion. Am J Orthod Dentofacial Orthop. 2000;117(1):1-9.

[12] Primozic J, Farcnik F, Perinetti G, Richmond S, Ovsenik M. The association of tongue posture with the dentoalveolar maxillary and mandibular morphology in Class III malocclusion: A controlled study. Eur J Orthod. 2013;35(3):388-93.

[13] Velásquez V. Mordida cruzada anterior: diagnóstico y tratamiento con placa progenie. Revista Latinoamericana de Ortodoncia y Odontopediatría [Internet]. 2005. Disponible en https://www.ortodoncia. ws/publicaciones/2005/art-8/

[14] Hernández JA, Padilla MR. Tratamiento temprano de la mordida cruzada anterior. Revisión de la literatura. Rev Estomat. 2011;19(2):40-7.

[15] Villegas AS. Relación esquelética clase III combinada por retrusión del maxilar superior y prognatismo mandibular con mordida cruzada anterior y posterior bilateral y hábito de protrusión lingual. CES Odontol. 2004;17(1):63-9.

[16] Raymond JL, Pimentel I, Vignolo R. Tratamiento ortopédico de las maloclusiones de clase III: masticación y plano oclusal. Ortodoncia Clínica. 2009;12(4):186-93.
[17] Haas AJ. Palatal expansion: Just the beginning of dentofacial orthopedics. Am J Orthod. 1970;57(3): 219-55.

[18] Wilmes B, Ngan P, Liou EJ, Franchi L, Drescher D. Early Class III facemask treatment with the hybrid hyrax and Alt-RAMEC protocol. J Clin Orthod. 2014;48(2):84-93.

[19] Nienkemper M, Wilmes B, Pauls A, Drescher D. Maxillary protraction using a hybrid hyrax-facemask combination. Prog Orthod. 2013;14(1):5. doi: http://dx.doi.org/10.1186/2196-1042-14-5.

[20] Wilmes B, Nienkemper M, Drescher D. Application and effectiveness of a mini-implant- and tooth-borne rapid palatal expansion device: The hybrid hyrax. World J Orthod. 2010;11(4):323-30.

[21] Franchi L, Baccetti T, Masucci C, Defraia E. Early Alt-RAMEC and facial mask protocol in Class III malocclusion. J Clin Orthod. 2011;45(11):601-9.

[22] Machado Cruz R. Eric Liou Interview. Rev Dent Press Ortodon Ortop Facial [internet]. 2009;14(5):27-37. Disponible en: http://www.scielo.br/scielo.php?script $=$ sci_issuetoc\&pid=1415-541920090005\&ln$\mathrm{g}=\mathrm{es} \& \mathrm{nrm}=1$.

[23] Jäger A, Braumann B, Kim C, Wahner S. Skeletal and dental effects of maxillary protraction in patients with angle Class III malocclusion. A meta-analysis. J Orofac Orthop. 2001;62(4):275-84.

[24] Sar C, Arman-Özçırpıcı A, Uçkan S, Yazici AC. Comparative evaluation of maxillary protraction with or without skeletal anchorage. Am J Orthod Dentofacial Orthop. 2011;139(5):636-49. doi: http:// dx.doi.org/10.1016/j.ajodo.2009.06.039.

[25] Ngan P, Yiu C, Hu A, Hägg U, Wei SH, Gunel E. Cephalometric and occlusal changes following maxillary expansion and protraction. Eur J Orthod. 1998;20(3):237-54. 